\title{
Educación superior para las juventudes rurales: reflexiones sobre el caso de la ingeniería en innovación agrícola sustentable, en Cuquío, Jalisco, México
}

\begin{abstract}
Resumen:
El artículo aborda una experiencia de educación superior en el medio rural, implementada en el municipio de Cuquío, en Jalisco, México. Para el análisis se enfoca en la Ingeniería en Innovación agrícola Sustentable, carrera que se ofrece en una zona con tendencias agroindustriales, que están teniendo efectos socioambientales considerables. Se reflexiona en torno a las implicaciones de la educación superior para las trayectorias de vida juveniles, y su inclusión tanto en el mundo laboral como en el agrícola, aportando a la discusión de la condición juvenil rural y los cruces con desarrollo rural.
\end{abstract}

Palabras clave:

Juventudes Rurales. Educación Superior. Agricultura.

\section{Educação superior para a juventude rural: reflexões sobre o caso da engenharia em inovação agrícola sustentável em Cuquío, Jalisco, México}

Resumo: $O$ artigo trata de uma experiência de educação superior em áreas rurais, implementada no município de Cuquío, em Jalisco, México. A análise enfoca a Engenharia em Inovação Agrícola Sustentável, uma carreira que é oferecida em uma área com tendências agroindustriais, que estão tendo consideráveis efeitos sócio-ambientais. Ela reflete sobre as implicações da educação superior nas trajetórias de vida dos jovens, e sua inclusão tanto no mundo do trabalho quanto na agricultura, contribuindo para a discussão da condição da juventude rural e das interseções com o desenvolvimento rural.

Palavras-chave: Juventude Rural. Educação Superior. Agricultura.

\footnotetext{
1 Investigador postdoctoral en la Maestría en Gestión y Desarrollo Social de la Universidad de Guadalajara, Financiado por el Consejo Nacional de Ciencia y Tecnología (CONACYT) de México. E-mail: mpsdavids@gmail.com. ORCID iD: https://orcid.org/0000-0002-8725-2053.

2 Profesora de tiempo completo en Instituto Tecnológico Mario Molina Aula Cuquío. E-mail: natalia.guerra@tala.tecmm.edu.mx. ORCID iD: https://orcid.org/0000-0002-3588-9183.
} 


\title{
Higher education for rural youth: reflections on the case of engineering in sustainable agricultural innovation in Cuquío, Jalisco, Mexico
}

\begin{abstract}
This article deals with an experience of higher education in rural areas, implemented in the municipality of Cuquí, Jalisco, Mexico. The analysis focuses on Engineering in Sustainable Agricultural Innovation, a career offered in an area with agro-industrial trends, which are having considerable socio-environmental effects. It reflects on the implications of higher education for the life trajectories of young people, and their inclusion in both the labor and agricultural worlds, contributing to the discussion of the rural youth condition and the intersections with rural development.
\end{abstract}

Keywords: Rural Youth. Higher Education. Agriculture.

\section{Introducción}

Lo reportado en este artículo forma parte de una investigación postdoctoral titulada Habitar y confrontarse en una zona de monocultivo de maíz: territorialidades y alternativas desde las juventudes rurales en el Instituto Tecnológico Superior Mario Molina de Cuquío. La investigación en general está orientada a comprender las formas como los jóvenes habitan en un contexto campesino que promueve la agroindustria como modelo de desarrollo rural, mientras que, paradójicamente, se forman profesionalmente para proponer proyectos de agricultura sustentable. La pesquisa se está desarrollando en contexto de la pandemia de covid-19, lo que reaviva el debate sobre la alimentación y el medio ambiente sanos como formas de prevención de ésta y otras enfermedades, en el marco más amplio de una crisis socioambiental generalizada, cuyos efectos son acumulables para las infancias y juventudes rurales.

Reflexionar sobre el acceso de las juventudes rurales a la educación superior y su experiencia al respecto es un tema urgente, ya que todavía en la actualidad la desigualdad entre las juventudes urbanas y las rurales es muy notoria. En Latinoamérica hay alrededor de 30,7 millones de jóvenes rurales (entre 15 y 29 años); de los cuales 11,9 millones no trabajan (estudian, trabajan en quehaceres domésticos) y 9,6 millones trabajan en el sector agrícola, en los cuales la incidencia de la pobreza es más alta (DIRVEN, 2016). En el caso de México 4 de cada 10 jóvenes en México viven en zonas rurales, y 6 de cada 10 jóvenes rurales viven en situación de pobreza y 2 de cada 10 en pobreza extrema (SOLOAGA, 2018).

El caso presentado es relevante debido a que la ingeniería que se analiza, puede ser un proyecto donde se crucen la incubación de alternativas de desarrollo rural y agricultura sustentable con la participación de las juventudes rurales, así como la generación de proyectos y políticas públicas que propongan acciones afirmativas que reduzcan la brecha de desigualdades de jóvenes en el medio rural.

El artículo está compuesto por varios apartados, en el primero se plantea la metodología que se está utilizando en el proceso investigativo. Posteriormente se aborda el contexto que se está indagando, presentando algunos datos generales del municipio; además se describe la institución que oferta la ingeniería para estas juventudes.

Como parte de la discusión teórica se presenta en otro bloque, algunos ejes conceptuales en torno a las juventudes rurales, la educación y el empleo, como parte de la condición juvenil rural, que permiten tener una lectura más profunda de la experiencia presentada.

En un siguiente apartado se plasma una exploración sobre la situación de estudiantes y egresados de la ingeniería, a través de algunos datos relacionados con un seguimiento a egresados. Además, se presentan algunos relatos de estudiantes y profesores sobre la ingeniería 
y su vinculación al mundo laboral. Estableciendo algunas relaciones con los conceptos presentados en el bloque anterior.

Se concluye con algunas reflexiones respecto a la necesidad de promover y potenciar procesos sociales desde las juventudes rurales, que sean críticos al estado actual de crisis socioambiental y que aporten alternativas para una agricultura sustentable.

\section{Metodología}

La obtención y el manejo de datos es parte de una estrategia de Investigación-Acción participativa (EMIRO, 2010), desde la que se convocó a la formación de un grupo de discusión integrado principalmente por estudiantes del Instituto tecnológico, un par de profesores y el facilitador externo.

La metodología es de corte cualitativo, privilegiando la observación participante etnográfica, el registro en diarios de campo y las entrevistas semiestructuradas a distintos actores clave.

Los datos están siendo analizados desde la perspectiva de la Condición Juvenil Rural (SÁNCHEZ, 2020), que es una propuesta de análisis de juventudes rurales que integra para su comprensión las dimensiones estructural, territorial e intersubjetiva para una comprensión de la complejidad en que se configuran las juventudes rurales del municipio.

\section{Sobre el contexto de Cuquío y el Instituto Tecnológico Mario Molina:}

Cuquío es un municipio rural del estado de Jalisco, ubicado en la región centro (IIEG, 2019). Según el último censo nacional el municipio tiene 17,820 habitantes (INEGI, 2020), de los cuales el 16\% (2867) se encuentran entre los 15 y 24 años.

\section{Ilustración 1 - Localización geográfica de Cuquío}

\section{LOCALIZACIÓN GEOGRÁFICA DE CUQUÍO (RESPECTO AL ESTADO Y AL PAÍS)}

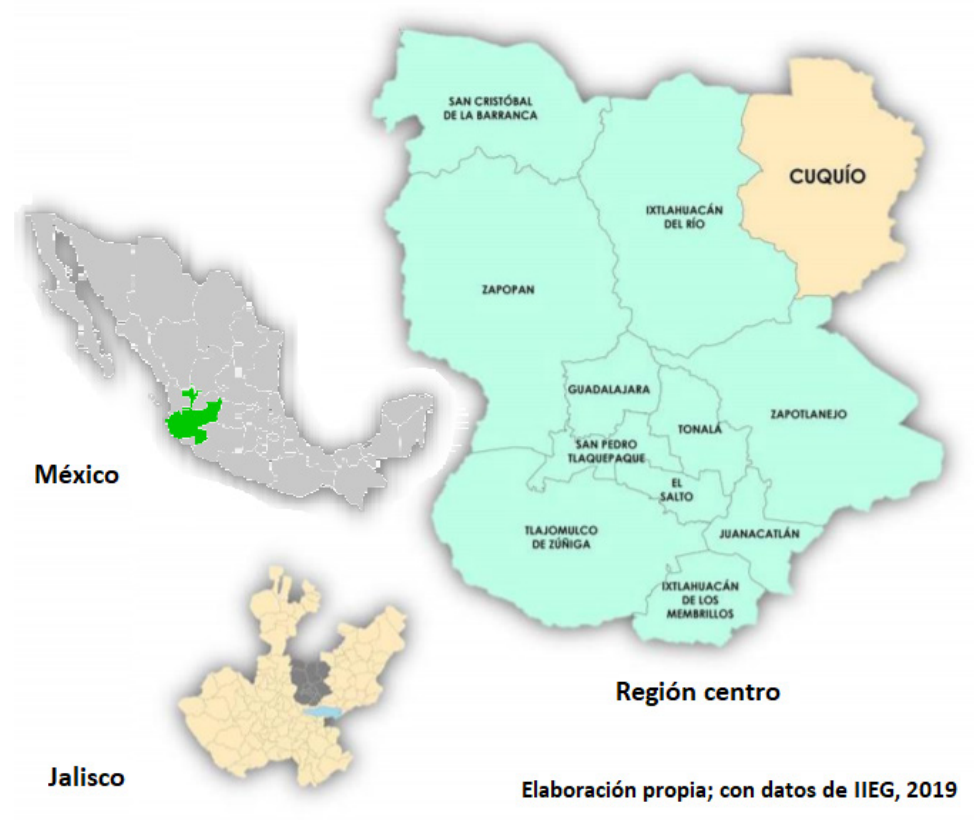

Fuente: Elaboración propia, con datos de IIEG, 2019. 
Histórica y regionalmente es conocido como El granero de Los Altos aludiendo a su vocación agrícola, y cuenta con un pequeño valle en el que tradicionalmente se llegó a sembrar trigo a inicios del siglo pasado, y sobre todo ha sido importante el monocultivo de maíz que se alterna con los "coamiles", que son pequeñas parcelas de autoconsumo, que utilizan sistema milpa (maíz, frijol calabaza), en una dinámica tensa entre la agroindustrialidad y la campesinidad.

En el municipio de Cuquío, en la década de los ochenta y la de los noventas,

se implementó un proceso de modernización y urbanización en diversos ámbitos societales, lo que derivó en organizaciones económicas conformadas por campesinos que pretendían funcionar como agroempresas, producciones agropecuarias de tipo intensivas y alta productividad a base de insumos exógenos y para su venta a la industria alimenticia, [...]. Sin embargo, esta urbanización y modernización, ha devenido la erosión de las solidaridades locales, [...] en una actividad agropecuaria y doméstica de alto consumo de petroinsumos y alta productividad maicera pero de insuficiente rentabilidad y alto impacto ambiental, $y$, principalmente, ha convertido a los habitantes del municipio en consumidores dependientes de los insumos exógenos, alimentos procesados por la agroindustria. (ESPINOSA, 2017, p. 116).

Hacia el 2015, en Cuquío el 56.5 por ciento de la población se encontraba en situación de pobreza, es decir 9 mil 285 personas comparten esta situación en el municipio, así mismo el 37.3 por ciento (6,121 personas) de la población es vulnerable por carencias sociales (IIEG, 2019). El municipio tiene un índice alto de migración a Estados Unidos y otras zonas urbanas del estado, principalmente a la capital, Guadalajara. En específico muchos jóvenes migran después de su educación de bachillerato para poder estudiar una carrera profesional.

La vida universitaria ha sido una opción creciente para las y los jóvenes de Cuquío en las últimas décadas. Con el aumento de escuelas de educación básica y media superior, la escolarización fue siendo vista como una trayectoria posible y deseable. Cada vez hay más jóvenes en la preparatoria, es común preguntar a estos, qué es lo que van a estudiar, o a que carrera harán trámites de ingreso. Así la mayoría de las y los jóvenes en los últimos años se están preguntando si estudiar o no estudiar, y al hacerlo se topan con la estructura y lo que realmente pueden hacer.

Estudiar en Guadalajara, la capital del estado, ha representado un esfuerzo considerable, tanto para la familia como para el joven. La primera opción casi siempre había sido intentar hacer trámites de ingreso a la Universidad de Guadalajara, por su carácter de pública y por su amplia oferta educativa, sin embargo, un primer obstáculo es la prueba de admisión, que evalúa las capacidades matemáticas y de lectura y comprensión, aspectos que generalmente son muy débiles en la educación básica en el medio rural, por lo que representa una desventaja acumulada (SARAVÍ, 2009).

Por otra parte el joven foraneo en Guadalajara, se enfrenta a otra sociedad, a las costumbres urbanas, a otros modos de vivir la vida; los desea en algún sentido pero le cuesta trabajo asimilarlos. La mayoría de universitarios con los que se ha tenido contacto mencionan una reafirmación de la identidad y una valoración de lo que daban como natural antes de ir a vivir a la ciudad. Estudiar en la ciudad implica muchos cambios para un joven rural. Esto comenzó a cambiar en 2010, cuando incursionó en Cuquío el Instituto Tecnológico Mario Molina. Esta opción alargó más el periodo juvenil de muchos jóvenes que por su situación económica no tenían la posibilidad de vivir y estudiar en Guadalajara, se habla de un alargamiento, porque lo más común es que los jóvenes que no estudian más allá de la preparatoria se casen o se unan en pareja, con lo que para la comunidad pierden el estatus de jóvenes.

El Instituto Tecnológico Mario Molina en Cuquío, es una extensión o aula a distancia de la Unidad Académica de Tala, en Jalisco. Es una opción de formación técnica que forma parte de la institución de educación superior tecnológica más grande de nuestro país, el Tecnológico Nacional de México (TecNM). ${ }^{1}$

1 Toda la información relacionada con el Tecnológico Nacional de México ha sido tomada y sintetizada de diferentes secciones de la página web de la institución: https://www.tecnm.mx/. 
En el marco de la educación tecnológica superior en México se encuentra que los primeros Institutos Tecnológicos (IT) surgieron en México en el año de 1948. En 1959, los Institutos Tecnológicos son desincorporados del Instituto Politécnico Nacional, para depender, por medio de la Dirección General de Enseñanzas Tecnológicas Industriales y Comerciales, directamente de la Secretaría de Educación Pública. Una estrategia de educación que permite tener un alcance paulatino de alcance a nivel nacional.

El 23 de julio de 2014, fue publicado en el Diario Oficial de la Federación, el Decreto Presidencial por el que se crea el TecNM. De acuerdo con el Decreto citado, el TecNM se funda como un órgano desconcentrado de la Secretaría de Educación Pública, que sustituye a la unidad administrativa que se hacía cargo de coordinar este importante subsistema de educación superior.

El Tecnológico Nacional de México está constituido por 254 instituciones, en las cuales se atiende a una población escolar de más de 600 mil estudiantes en licenciatura y posgrado en todo el territorio nacional. Convirtiéndola entonces en la Institución de educación Superior más grande a nivel nacional.

Como parte del Tecnológico Nacional de México y de su presencia en el estado de Jalisco, se encuentra fundado desde el 23 de agosto del 2016, el Instituto Tecnológico José Mario Molina Pasquel y Henríquez (TecMM $)^{2}$, el cual tiene la finalidad de prestar servicio de educación superior tecnológica en el Estado de Jalisco, adscrito al Tecnológico Nacional de México y sectorizado a la Secretaría de Innovación Ciencia y Tecnología. Teniendo como encargo responder a los objetivos del Plan Nacional de Desarrollo y del Plan Estatal de Desarrollo de Jalisco, que establece el compromiso de un "México con educación de calidad". Por lo que se unifican 13 Institutos Tecnológicos Superiores, en un solo Organismo Público Descentralizado, con una Dirección General y 13 Unidades Académicas en el Estado de Jalisco. Con el propósito de eficientar la estructura académica y administrativa.

Esta red de trece unidades académicas y doce extensiones, tiene un alcance de Educación Superior en once de las doce regiones del Estado de Jalisco con más de 15,000 estudiantes, esto contempla el 9.74\% de la matrícula de Educación Superior, con 16 ingenierías, 5 licenciaturas, y 2 posgrados.

Para efectos de este artículo se considera la unidad académica de Tala y su extensión Cuquío, la cual tiene la siguiente oferta académica:
a. Ingeniería en Administración
b. Ingeniería en sistemas
c. Ingeniería en Innovación Agrícola Sustentable

La extensión se encuentra en funciones desde Agosto 2010, en todo este tiempo han egresado 2 generaciones de Ingeniería Industrial, 6 de Ingeniería en Administración, 1 de Ingeniería en Sistemas y 5 de Ingeniería Innovación Agrícola Sustentable. En esta institución trabajan 16 docentes, 2 administrativos y estudian 131 alumnos.

El análisis que se presenta solo considera la ingeniería en Innovación Agrícola Sustentable; pues se considera la más exitosa de esta institución en el municipio de Cuquío, por estar íntimamente ligada con la vocación agrícola del mismo. Esta ingeniería tiene como objetivo: "Formar profesionistas analíticos y críticos, comprometidos socialmente y con sólida cultura científico tecnológica, que les permita la planeación del desarrollo regional en el contexto de la sustentabilidad, para realizar investigación, validación, transferencia, adaptación, producción e innovación agrícola”. Como se mencionaba anteriormente, uno de los aspectos más interesantes de esta ingeniería es la tensión entre la intención de agricultura sustentable que declara el Instituto tecnológico como objetivo de la

\footnotetext{
2 Toda la información relativa al Tecnológico Mario Molina, ha sido recuperada de la página http://tecmm.edu.mx/ y sintetizada para fines de presentación.
} 
formación y el contraste con la promoción de la agroindustria en el estado de Jalisco y el municipio, que genera algunas dinámicas de exclusión social, además de los efectos ambientales.

\section{Algunas categorías para pensar la condición juvenil rural y la educación superior en un contexto de expansión agroindustrial}

Los estudios sobre la juventud rural comenzaron en la década de los noventa del siglo pasado, relacionados con las temáticas de los actores rurales para el desarrollo. Se identifican tres grandes áreas en estos estudios:

La primera, los que se refieren a la educación y capacitación de los jóvenes rurales para el desarrollo; la segunda los que abordan la temática de la incorporación de la juventud rural al trabajo, y la tercera tiene que ver con la implementación de políticas específicas para la juventud rural. (PACHECO, 2013, p. 21).

Siguiendo otras revisiones del estado del arte, la relación entre juventud rural, educación y desarrollo rural está presente en la mayoría de los estudios (KESSLER, 2006). El desarrollismo en lugares como Cuquío,

además de un discurso hegemónico, se convirtió una apuesta estatal materializada en distintas intervenciones estructurales, como la migración institucionalizada a Estados Unidos, la producción de maíz como agronegocio y la educación hasta la profesionalización, estas fueron apoyadas de distintas maneras por políticas y programas estatales hasta que se constituyeron como las principales trayectorias para las juventudes en el medio rural. Después el mismo Estado mexicano gira hacia el neoliberalismo, y va desestructurando lo que apoyaba dichas trayectorias. (SÁNCHEZ, 2020, p. 8).

El aumento de la escolarización en los países tiene una relación directa con la condición juvenil (MORCH, 1996). En el discurso político-ideológico del Estado, a lo largo de todo el siglo pasado el valor de la educación fue reconocido como el principal instrumento para la construcción de lo nacional y para alcanzar el progreso y la modernización (MESEGUER, 2012). Desde esta lógica, poco a poco se fue garantizando el acceso a la educación básica en gran parte de las zonas rurales del país, y posteriormente el gobierno fue promoviendo la escolarización como una vía de ascender en la movilidad social, puesto que

la inversión en la educación de las generaciones juveniles rurales era un camino que podía acortar la legitimación de los técnicos de la extensión agrícola y acelerar los procesos de transformación tecnológica. La juventud se volvía el punto de partida de la promoción de cambios sociales y tecnologías necesarias al desarrollo del capitalismo en el espacio agrario europeo. Socializarla, en instituciones educativas formales y no formales, era una cuestión de desarrollo socioeconómico. (BEVILAQUA, 2009, p. 231).

Respecto a la relación entre juventud y la educación como política pública, el informe: "Políticas de inclusión social de jóvenes en américa latina y el caribe: Situación, desafíos y recomendaciones" (ALVARADO; RODRIGUEZ; VOMMARO, 2013), hace una revisión de políticas públicas de juventud, extensa, tomando el análisis de 10 países, y formulando algunas reflexiones articuladas con los saberes académicos. Todo lo detallado del informe se puede resumir muy bien esta frase "en el contexto latinoamericano, las políticas sociales y publicas se restringen para los jóvenes - en el mejor de los casos - al ámbito de la educación formal o capacitación, a la salud y 
al deporte" (REGUILLO, 2012, p. 125). A lo largo del informe se puede notar la ausencia del tema ambiental y de la producción de alimentos sanos, tanto en las políticas públicas revisadas, como en las reflexiones académicas expresadas.

En el informe se justifica muy bien el necesario aumento al acceso a la educación, y se enumeran algunos problemas presentes en el dispositivo escolar; pero no se cuestiona de fondo el carácter reproductor de la escuela (BORDIEU; PASSERON, 2003), sobre todo en este contexto de crisis. Se explican a fondo los abordajes necesarios para atender la salud de las juventudes, poco se habla de que la salud va ligada a la alimentación y que los alimentos actuales propician la aparición de enfermedades crónico-degenerativas, fuerte problema asociado con la falta de seguridad social y el previsto "envejecimiento" de la población latinoamericana en las siguientes décadas. Se sigue hablando de empleo y de mejorar las condiciones de este, pero no se habla de la estructura jerárquica implícita en el empleado y el empleador, y que por mucho que mejore el empleo la jerarquía presente alimenta la desigualdad. Menos aún se habla del trabajo para la subsistencia y producción de los alimentos propios en contextos rurales, y el papel que tiene el territorio en las aspiraciones de inclusión económica de los jóvenes (CAZZUFFI; DÍAZ; FERNÁNDEZ; TORRES, 2018).

El modelo agroindustrial propuesto desde las estrategias de desarrollo primero y de neoliberalización después (KAY, 2019), ha generado la contaminación de todo el ambiente, ha propiciado el acaparamiento de tierra, ha desaparecido opciones de trabajo para millones de campesinas y campesinos, ha despojado a la agricultura de su significado profundo de vida, y la ha querido convertir en negocio (DETSCH, 2018). El resultado son millones de hombres y mujeres expulsados del campo, jóvenes que desean vivir en ciudades imaginarias con estilos de vida insostenibles, y que al emigrar a ellas llegan a urbes plagadas de problemas, y tardan tiempo en darse cuenta que han caído en una trampa, de la que luego da miedo salir.

Con dicha situación, y la poca calidad educativa que existe en el campo (MESEGUER, 2012), es posible entender que las desigualdades desde lo escolar para las juventudes rurales son mayores, pues "las situaciones más extremas de exclusión son el resultado final de procesos de acumulación de desventajas" (SARAVÍ, 2009, p. 23). Un joven que pasa tres años en una escuela que no lo forma con una calidad mínima aceptable, es un joven al que se le irá complicando la demás trayectoria escolar; y al ser el nivel educativo "uno de los principales activos que permitirá completar [sus transiciones de vida] en mejores o peores condiciones" (SARAVÍ, 2009, p. 73); entonces enfrentará una carrera en desventaja. De esta manera la educación "persiste en su función reproductora del sistema social, propiciando una reafirmación de sus estructuras y dinámicas actuales. Así la escuela se constituye en una institución socializadora transmisora de la cultura imperante" (FHURER, 2009, p. 56).

La desigualdad en la educación entre campo y ciudad ha sido un tema abordado siempre que se habla de jóvenes rurales. "Hay una profunda desigualdad en los conocimientos adquiridos al finalizar la escuela secundaria por un joven o una joven que haya asistido a una escuela privada, a una pública, o al sistema de telesecundaria" (SARAVÍ, 2009, p. 209). Por lo es evidente que, estructuralmente, la trayectoria educativa está atravesada por la desigualdad y la acumulación de desventajas en la juventud rural, al querer acceder a la universidad. "La influencia del origen social perdura a lo largo de toda la escolaridad y se hace especialmente sensible en los grandes virajes del recorrido escolar" (BORDIEU; PASSERON, 2003).

Actualmente a pesar de que la escuela y el trabajo han representado tradicionalmente mecanismos clave de movilidad e integración social, "los jóvenes de los sectores populares no ven en la escuela una alternativa real y tangible de movilidad social; la escuela carece de capacidad de interpelación sobre los sujetos" (SARAVÍ, 2009, p. 248). Si se logra entrar a la universidad, además de que poderla concluir es una competencia de obstáculos, al salir el joven rural, al igual que todos los demás debe enfrentar "la frustración de expectativas generadas porque los egresados no logran insertarse laboralmente" (KESSLER, 2007, p. 31). Aun teniendo trabajo se enfrentará a la situación de precarización laboral de los profesionistas, producto de todas las transformaciones socioeconómicas que han impactado los mercados de trabajo, especialmente en el medio rural. 
Por todo lo anterior es necesario, "generar conocimiento sistemático del sujeto social y su problemática específica [...] habida cuenta de la amplia heterogeneidad que la caracteriza, constituye una base necesaria para relevar su problemática" (ESPEJO, 2017, p. 9).

Las dinámicas que llaman a la urgencia en la inversión en juventud se vuelven aún más complejas si se tienen en cuenta factores como la pertenencia étnica, el género y el área de residencia de las y los jóvenes en América Latina y el Caribe. La reproducción de la pobreza y su incidencia entre ellas y ellos se amplifica al considerar estos factores. El pacto demandado, por tanto, no es sólo intergeneracional, sino también multicultural y de género. Abordar ahora la naturaleza de las múltiples exclusiones en el acceso a servicios, empleo y participación política y social que afectan a las y los jóvenes que pertenecen a pueblos indígenas y/o afrodescendientes y/o habitan en áreas rurales, y a las mujeres jóvenes, resulta esencial para la consolidación democrática y de los principios de la ciudadanía en nuestras sociedades. (NU; CEPAL, 2012).

\section{Análisis de la experiencia y discusión de resultados parciales}

Para abordar el caso de la ingeniería en Innovación Agrícola sustentable, planteamos dos vías, la primera haciendo una revisión de los egresados de la carrera, lo cual nos permite tener algunos datos específicos para comenzar a ver el impacto que ha tenido en el municipio. Posteriormente para darle sentido a estos datos cuantitativos, presentamos algunos testimonios de egresados y un profesor que ha participado en esta carrera, lo cual nos dará una aproximación cualitativa a la experiencia de esta opción de educación superior para las juventudes rurales.

Es necesario recordar que es una investigación en curso y se siguen analizando más datos que se están obteniendo en el proceso, por lo que este artículo más que presentar datos empíricos ya completamente trabajados, lo que pretende es proponer algunas pistas para seguir el debate de la educación superior para juventudes rurales.

\section{Seguimiento de egresados}

Desde su apertura en el año 2010 del aula a distancia Cuquío del Instituto Tecnológico hasta el 2020, se han tenido 5 generaciones de egresados de la carrera de Innovación Agrícola Sustentable.

En la siguiente gráfica se muestran los ingresos y egresos, de forma global hombres y mujeres con datos proporcionados por la institución:

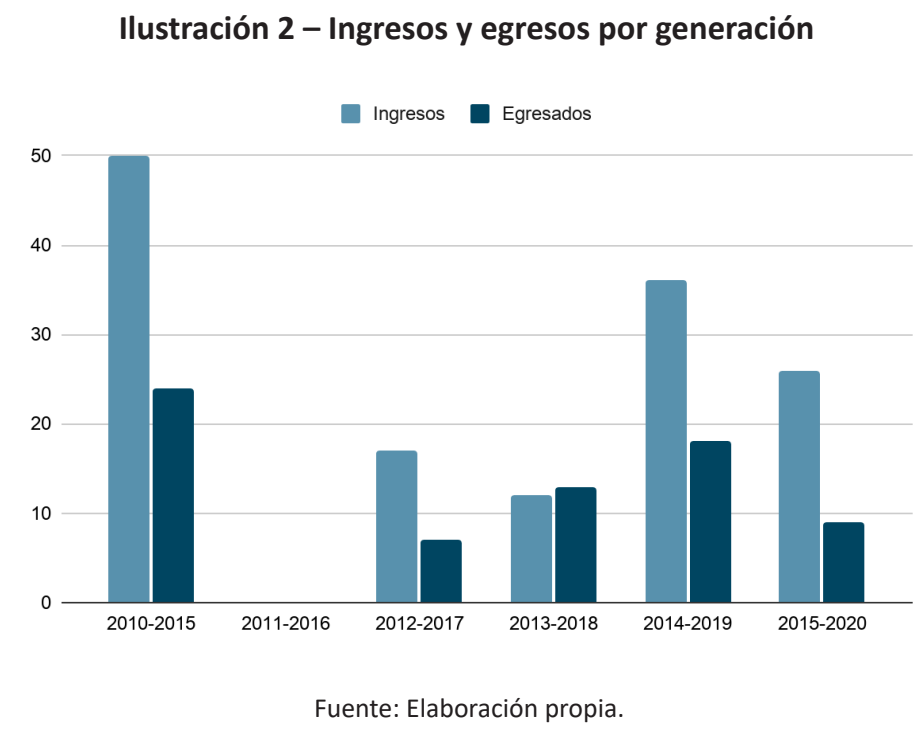


Respecto a la generación 2011, no se brindó la carrera porque no hubo demanda de estudiante; para este análisis de considera la segunda generación hasta el 2012, en algunas generaciones hay variaciones respecto a los egresados con el grupo de origen, ya que se integran alumnos con rezago para culminar.

Mostrándose en porcentajes respecto ingreso y egreso, por medio de la regla por tres simple. Donde la primera generación tiene un egreso del $48 \%$, segunda $41 \%$, tercera $108 \%$, cuarta $50 \%$ y finalmente la quinta $35 \%$.

\section{llustración 3 - Porcentaje de ingreso y egreso}

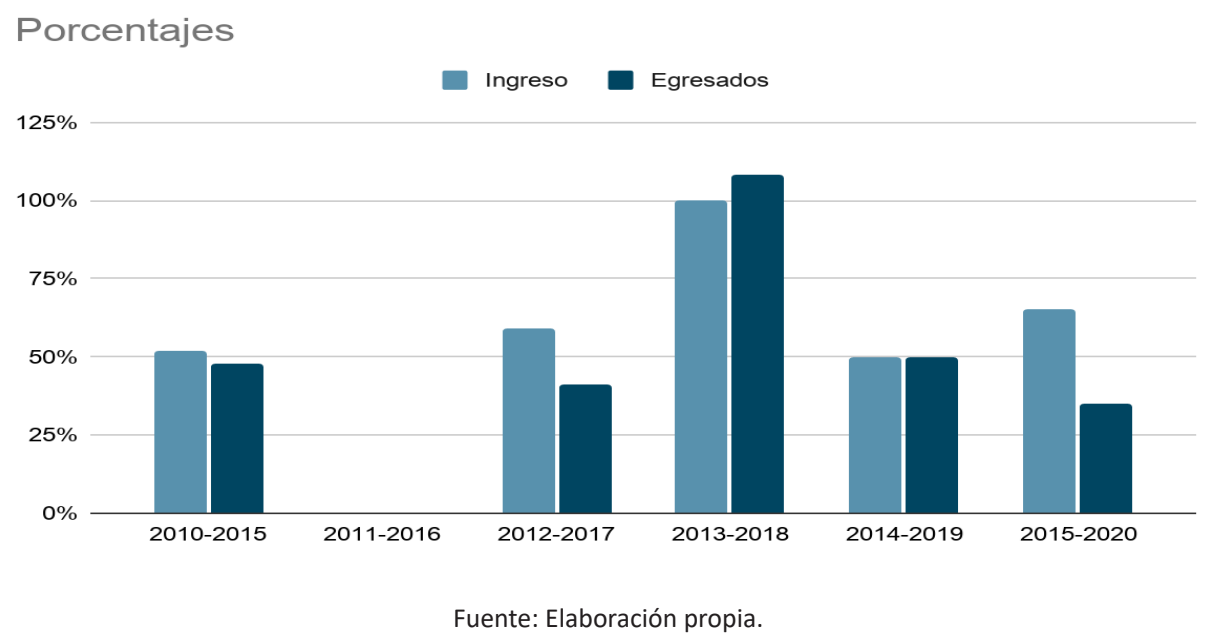

En cuanto al egreso de mujeres $(\mathrm{M})$ respecto a hombres $(\mathrm{H})$, tenemos primera generación $11 \mathrm{M} / 13 \mathrm{H}$, segunda $\mathrm{M} 3 / \mathrm{H} 4$, tercera $8 \mathrm{M} / 5 \mathrm{H}$, cuarta $6 \mathrm{M} / 12 \mathrm{H}$, quinta $3 \mathrm{M} / 6 \mathrm{H}$.

\section{llustración 4 - Comparativa de egresos hombres y mujeres}

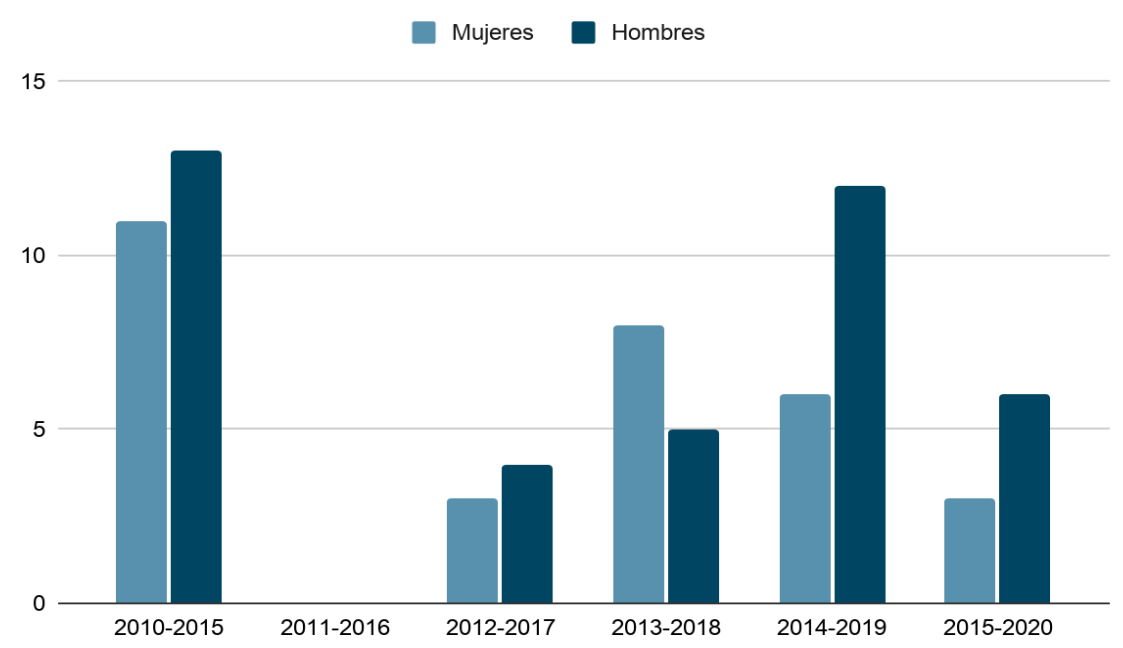

Fuente de datos: Elaboración propia, con datos de IIEG, 2019.

\section{Aproximaciones a los discursos de los involucrados}

Para el objetivo de este artículo se consideraron algunas entrevistas semiestructuradas realizadas a egresados de la ingeniería y a profesores que han impartido clases en la carrera. Por 
la extensión del artículo y las reflexiones que en éste se plasman se decidió hacer una selección de dos experiencias de estudiantes, un hombre y una mujer, y una de un profesor que ha tenido participación constante en las distintas generaciones. Se transcribieron algunos fragmentos del total de la entrevista y se exponerlos editados a modo de relato en conjunto, para no perder el sentido de las ideas. A continuación se presentan los relatos, en los cuales se encuentran subrayados algunos elementos que sobresalen para el análisis tomando en cuenta las discusiones presentadas en el apartado anterior.

Relato de egresado Masculino:

Mi primer trabajo al salir de la carrera fue en la empresa Gruma S.A de C.V., mejor conocida como MASECA, ese trabajo si está relacionado con mi carrera, yo trabajaba en un puesto llamado analista de embarques, dicho empleo recibía lo de $\$ 12$ pesos mensuales, más pagos de viáticos, en esta empresa no trabajas en sitio fijo, si no que te mandan a distintos municipios donde están ubicadas las bodegas, que le venden su producto a MASECA, que compran el maíz a los productores y este a su vez se lo vende a MASECA, pasando por la mayor parte del Estado de Jalisco. [...] Mi experiencia personal te podría decir que al principio no me sentía preparado, porque era un área donde no había trabajado, más bien en la escuela era másteoría, nada que ver con lo que en ese puesto se desempeñe, mi experiencia personal te podría decir que con el tiempo y la práctica vas aprendiendo a realizar tu trabajo correctamente, te dan capacitaciones y te ponen a practicar y practicar hasta que lo haces bien, y ya te dejan solo, tienes a alguien que te enseña un par de semanas, antes de dejarte solo en el trabajo, pero si ayuda el estudio. [...] Actualmente yo trabajo para un programa gubernamental, se llama SEGALMEX (Seguridad Alimentaria Mexicana) y también está relacionado con la carrera. Aquí me pagan 15,000 mensuales y estoy trabajando en el municipio de Unión de Tula, y ahora te podría decir a comparacion de mi primer empleo, me siento muy capacitado y capaz para desempeñar mi trabajo y mi puesto, porque ya traigo arrastrando experiencia de los trabajos anteriores, ya traigo una experiencia de unos 5 años, si me siento con el conocimiento necesario para cubrir este puesto.[...] Si pienso en los obstáculos, si hay incertidumbre, la cuestión es que no sabes si habrá una vacante para empezar a poder trabajar y desarrollarte profesionalmente, no sabes en tu municipio o no, que es lo primero que uno busca estar cerca de tu casa, también bien puede ser que el que tenga más diplomas, el que tenga más títulos, pero ya en el campo es muy diferente, ya no es estar en un aula, ya es tratar estar directamente con los productores, $\mathrm{y}$ si no te desenvuelves bien en el ámbito profesional, puedes terminar ser un simple jornalero y trabajar en el campo y de nada sirve la carrera o también estudiar por estudiar y para no ejercer, terminas ayudando a tu papa y quedándote en tu casa por comodidad. [...] Considero que el aprendizaje que obtuve fue el adecuado, por el hecho de que el estudio te abre puertas, solo es cuestión de estar ahí, buscándole, tocándolas, pero si, fue lo adecuado lo que necesitaba para trabajar profesionalmente. [...] Siento que me faltó, tener mi título ya que solo tengo mi carta pasante, ya que solo al momento me he integrado al mundo laboral, pero si me falta eso que comprobar, aunque presento mi carta de pasante. [...] Del tiempo que estuve trabajando en MASECA, entré en una empresa local de Cuquío, estuve trabajando como año y medio, donde aprendí bastante, cosas que no aprendí en un aula, en un aula no te enseñan sobre de ingredientes activos de ciertas medicinas utilizadas en la producción de maíz por ejemplo, me tuve que enseñar a platicar con la gente, a tener un trato, para poder vender, ahí mi puesto era de vender agroinsumos; entras con miedo, no sabes hablar, no sabes vender, no sabes qué productos están vendiendo, tienes que estudiar los productos y dar una buena recomendación, aparte de agroinsumos, semillas vendía tractores. Me ha servido cada empleo, es difícil hasta que te involucras, y pasa de ser difícil hacer algo constructivo, a ser un aprendizaje que te da valor como ingeniero. [...] De mis compañeros de generación, salimos 7 , sé que los hombres algunos trabajan con sus papás, en el campo, sé que algunas mujeres 2 se casaron y no trabajan, otra trabaja en el DIF, no ejerce la carrera, ironías de la vida soy el único que no se tituló. (Entrevista a Joven Egresado, 2021). 
Relato de egresada femenina:

Mi trabajo al salir de la escuela, alrededor de dos años, fue dedicarme a mi familia, que también es trabajo, porque salí embarazada. Posteriormente estuve trabajando en el negocio de mis papás, ya me lo pasaron a mí y por la pandemia ya no seguí, ya va ser un año que deje, pero por suerte este año pasado, en marzo en abril, entre a trabajar en un trabajo relacionado a mi carrera. [...] Estoy trabajando en lo que es de mi carrera, antes se llamaba BEDR Brigada de educación para el desarrollo rural, ahora se llama UEMSTAyCM (Unidad de educación media superior tecnológico agropecuario y ciencias del mar), no me sentía tan preparada para eso, es darle capacitación a comunidades rurales, pero poco a poco me fui integrando. Estoy con un profe, ahora soy su compañera, con ayuda de él y mi jefa y compañeros me estoy adaptando y lo bonito, que es aquí mismo en mi municipio, y es donde yo hice mi servicio social y mi proyecto de titulación, también por eso me conocieron y tuve la oportunidad de quedarme ahí. Me pagan alrededor de \$3500 la quincena, con todas las prestaciones de ley, tengo seguro y si estoy muy contenta con eso. [...] Los principales obstáculos al salir la carrera fue el que no me sentía capaz de hacer un huerto, como que creo que me hizo falta más práctica, sentía que a lo mejor no encajaba en ningún trabajo, necesitaba como más experiencia con los agricultores, ya ahorita le estoy agarrando esa experiencia, poco a poco ya estoy creyendo más en lo que aprendí. [...] La educación recibida en el Tecnológico, en lo teórico se me hizo que era adecuada, por hay mucha herramienta y libros en internet, si realmente quieres buscar y te interesa, no necesitas que el mismo maestro te lo esté poniendo, tu puedes investigar, pero si algo deficiente e insuficiente en la parte práctica, al menos cuando yo estuve, a nosotros nos tocó hacer unos invernaderos, y empezar hacer unos terrenos, pero si hubiera estado antes, hubiera estado antes, a lo mejor hubiéramos practicado más, tomado la experiencia como yo que comunidades que ahorita estamos visitando y me preguntaban de unas cosas de algunas cosas de algunos cultivos y yo me quedaba como eso me faltó [...], es lo que hace falta y es cuando realmente estás aprendiendo, yo ahorita lo he notado en mi trabajo, que estoy aprendiendo otra vez. [...] Lo que yo siento que falto para integrarse en el mundo laboral, lo mismo que dije anteriormente la práctica, tener mayor seguridad en el manejo de los cultivos, al menos dominar uno o dos, estar más relacionado, quiero pensar que se omitieron ciertas prácticas, porque algunos de mis compañeros ya tenían ranchos y se dedicaban a eso, porque ciertas prácticas en sus cultivos de maíz, para ellos ya eran común, y para mí no, yo nunca he de piscar, yo nunca he de moler, entonces ese tipo de cosas yo quería hacerlas, y nunca me lo enseñaron. Lohubieran hecho, no me interesa si nunca lo has hecho, con el maíz tal paso va primero, el segundo y tercero, e ir aprendiendo de los demás, y la parte de cómo debe de ser. Me faltó para poder integrarme y poder platicar con los dueños de rancho $y$ agricultores. (Entrevista a joven egresada, 2021).

\section{Relato de profesor de distintas generaciones:}

Desde que yo inicie en el Tecnológico fue en el 2011 ya llevaban un año, me tocó entrar con los de tercero, la primera generación, inicialmente había muchas personas que habían entrado a esa carrera [...] había muchas personas rezagadas, que querían tener una carrera, tener un título, pero dentro de esos alumnos que estaban ahí, podemos rescatar varios alumnos muy buenos, ejemplares, que ahorita están ahí trabajando, incluso en empresas propias. [...] La carrera como que en un principio no les llamó mucho la atención, que hasta de hecho un año no hubo alumnos [2011], no se completó el grupo y después de ese año, comenzaron, cuando más se incrementó fue cuando salió la primera generación y vieron que comenzaron a trabajar sin problemas. Entonces a partir de ahí, empezó a haber más afluencia e interés, personas que estudiaban en la UdG en Tepatitlan, la de agroindustrial, se regresaron para calarle, y aquí se quedaron. Está el caso de un alumno, de Palo Alto es un alumno que tiene su empresa propia, Ingeniero titulado, fue mi asesorado, en la etapa de residencia profesional y está trabajando. Así como él hay varios trabajando, tanto hombres como mujeres siguen trabajando, hay mucho trabajo para los Ingenieros del Tecnológico, se están abriendo las puertas. Ahorita me están pidiendo ya residentes, entonces ahorita los alumnos que están ingresando a octavo ya los están recomendando para que inicien a entrar a una empresa para que puedan hacer sus prácticas profesionales. Yo creo 
que la carrera de Innovación Agrícola ha funcionado bien el municipio, creo que es la que más ha funcionado, a mi punto de vista, ya que esta región es agrícola, entonces los alumnos se han incorporado aquí en las empresas que existen en la región y también fuera de ella, muchos se han ido fuera a estudiar posgrados, están otros trabajando en empresas ya de otro nivel y siguen trabajando. Creo que hace falta más investigación, necesitamos más investigación y que los alumnos le tengan más amor, porque como que le tienen miedo. El miedo es muchas veces porque no se les da las herramientas necesarias, aunque los muchachos lo hacen dentro de las empresas, y en la escuela hace falta reforzar más. (Entrevista PB, 2021).

Como se puede observar en estos relatos, existen distintas condicionantes que dificultan o facilitan las trayectorias escolares y laborales de las y los jóvenes rurales en Cuquío que pueden acceder a la educación superior brindada por el TecMM. Sobresalen las cuestiones de género, por ejemplo el hecho de que las mujeres egresadas pueden posponer su trayectoria laboral por la presión de atender a su pareja o a los hijos.

También aparecen cuestiones del mercado laboral, pues el estudio por sí mismo "no asegura esquivar las condiciones laborales que establecen una creciente precarización de las condiciones del trabajo asalariado de las juventudes rurales" (FHURER, 2009, p. 55). Quizá a eso se refiere el egresado cuando menciona la incertidumbre del desempleo, ya que "los jóvenes rurales, al tener un mayor nivel de calificación que sus padres, tienen escasas oportunidades de empleos de alta productividad en su zona de residencia, donde la demanda de trabajo es menos calificada y muchas veces estacional (GUISKIN, 2019). O quizá quedarse en el mismo municipio, como vendedores de agroinsumos que generan dependencia de los campesinos y problemas ambientales, a pesar que su formación profesional declare que buscan una agricultura sustentable.

Aparecen también cuestiones sobre relevo generacional en lo agrícola. El varón menciona que muchos de sus compañeros laboran con sus papás, lo cual muchas veces no les permite tener otro desarrollo laboral, quizá por el talante adultocéntrico de las relaciones intergeneracionales. Aunque también la egresada menciona un caso de éxito, cuando refiere que sus padres le transfirieron su negocio; lo cual nos recuerda que es necesario pensar de manera urgente las relaciones intergeneracionales.

Un aspecto que se repite en estas y otras entrevistas es la falta de práctica en la formación, lo cual es paradójico dado que la carrera se inserta en un contexto agrícola, lo cual sugiere que hacen falta alianzas estratégicas con productores de la región para que las y los jóvenes se integren en los distintos espacios productivos del municipio desde su periodo formativo, sin tener que espera a la graduación para poder buscar empleo. Por otra parte, respecto a la pertinencia de la formación es necesario "desarrollar competencias en las Instituciones educativas que generen motivación y empatía a los jóvenes del sector rural para realizar actividades de emprendimiento que forme en ellos la capacidad de crear conocimiento, aprender por sí solos, solucionar problemas y sean constructores de un futuro eficaz" (HERRERA; JHON, 2020, p. 103).

No se percibe en las entrevistas presentadas en este documento, ni en las que se han realizado con otros estudiantes, que haya una perspectiva de trabajo colectivo y organizado, con perspectiva emprendedora. Sigue siendo una visión muy anclada la fórmula de estudiar = encontrar trabajo, cuando la sustentabilidad es un tema que requiere más bien de una visión crítica y propositiva, debido a que la tendencia de la agricultura es al agronegocio y no a la sustentabilidad.

Sin embargo, es visible que el hecho de acceder a una carrera profesional le abre otro panorama a las y los jóvenes rurales que lo pueden hacer. Esto para las vidas concretas de algunos jóvenes es importante, ya que

En la agricultura familiar se encuentran trabas en el acceso a la tierra, tanto para ser propietarios como arrendatarios, con dificultades para acceder a créditos. Respecto a empleos rurales no agrícolas, se identifica una escasa atención de parte de las políticas públicas, lo que dificulta una inserción social exitosa en esta área. En cuanto al empleo por cuenta propia, se identifica la escasez de programas de capacitación e infraestructura. (GUISKIN, 2019, p. 27). 
Con las entrevistas realizadas hasta ahora en el proceso de investigación, más la revisión documental de datos de municipio que aparecen en el apartado de conceptos, podemos entender la tensión de promover la educación como trayectoria deseable, sin que necesariamente sea una educación anclada en el contexto y territorio (RIMISP, 2020). Por lo que es necesario trabajar en

las estructuras de oportunidades de los territorios que permitan vincular adecuadamente los activos y capacidades de los jóvenes, y que hagan posible a los sujetos percibir que pueden efectivamente insertarse laboralmente. Por ello, es importante que la acción pública combine inversiones en los individuos y en el territorio, para que sea posible desplegar el potencial de los jóvenes rurales, ya sea en el mismo lugar o en otro, si así fuera, por elección y no por obligación. (CAZZUFFI; DÍAZ; FERNÁNDEZ; TORRES, 2018, p. 21).

\section{Conclusiones}

En general se sigue pensando a los jóvenes rurales, solamente como una población vulnerable, que mejorará su situación únicamente con educación y empleo sin cuestionar de fondo las dinámicas que ocurren desde la dimensión estructural y que vienen modificando de manera profunda la vida en el medio rural. Cuando pensamos en términos de la condición juvenil rural e incluimos la dimensión intersubjetiva que le da sentido y significado a las vidas de los jóvenes y que se establece a través de las interacciones intergeneracionales, la mirada se hace más compleja.

Por lo anterior, cuando pensamos en la ingeniería analizada vemos necesaria una evaluación de impacto a profundidad de esta ingeniería que vaya más allá de la mirada tradicional, a fin de considerar mejorar y replantear la acción de esta opción educativa para los jóvenes rurales, atendiendo aspectos intersubjetivos y culturales, que ayuden a las y los jóvenes a ser propositivos en su contexto rural gracias a su formación, y no solamente a ser receptores pasivos de un programa académico que los forma para el trabajo en un contexto como el de Cuquío, cada vez más dominado por el modelo agroindustrial que está ocasionando fuertes problemas socioambientales y que tiene altas probabilidades de integrar a los jóvenes como una pieza más del sistema agroindustrial y no como impulsores de alternativas sustentables.

La apuesta de quienes esto escribimos es acompañar integralmente a las juventudes rurales que ingresan a este sistema educativo, para que asuman este momento de su formación como una posibilidad de incidencia y de transformación de las condiciones en que viven, lo cual implica reconocer a las y los jóvenes como agentes necesarios en los procesos de desarrollo de sus comunidades, o mejor aún, como sujetos sociales que puedan proponer alternativas al desarrollo desde sus contextos, problemáticas y necesidades.

\section{Referencias}

ALVARADO, Sara; RODRIGUEZ, Ernesto; VOMMARO, Pablo. Politicas de inclusión social de jóvenes en américa latina y el caribe: situación, desafíos y recomendaciones. Buenos Aires: CLACSO, 2013.

BEVILAQUA, Joel. Juventud rural: una invención del capitalismo industrial. Estudios Sociológicos, v. 27, n. 80, p. 619-653, mayo/ago. 2009.

BORDIEU, Pierre, PASSERON, Jean-Claude. Los herederos: los estudiantes y la cultura. Buenos Aires: Siglo XXI, 2003.

CAZZUFFI, Chiara; DÍAZ, Vivian; FERNÁNDEZ, Juan; TORRES, Javiera. Aspiraciones de inclusión económica de los jóvenes rurales en América Latina: el papel del territorio. Santiago: RIMISP, 2018. 
DETSCH, Claudia. La transformación social-ecológica del sector agrario en América Latina. México: Friedrich-Ebert-Stiftung; Proyecto Regional Transformación Social-Ecológica, 2018.

DIRVEN, Martine. Juventud rural y empleo decente en América Latina. Santiago, Chile: FAO, 2016.

EMIRO, Edgar. Investigación Acción. Metodología Transformadora. 2. ed. Zulia: Fondo Editorial UNERMB, 2010.

ESPEJO, Andrés. Inserción Laboral de los Jóvenes Rurales en América Latina: un breve análisis descriptivo. Grupos de Diálogo Rural, una estrategia de incidencia. Santiago, Chile: RIMISP, 2017.

ESPINOSA, Manuel. Estrategias rurales de reproducción social. La agroindustrialidad y la campesinidad de una organización en Jalisco, México. Guadalajara: Universidad de Guadalajara, 2017.

FHURER, Ariel. Juventudes rurales, educación superior y trabajo: anhelos y demandas para una inclusión social. Santiago Chile: Memoria para pregrado en Antropología social. Universidad de Chile, 2009.

GUISKIN, Maia. Situación de las juventudes rurales en América Latina y el Caribe. Ciudad de México: CEPAL, 2019.

HERRERA, Dolly; RIVERA, John. La Educación rural: Un desafío para la transición a la Educación Superior. Revista de Estudios y Experiencias en Educación, v. 19, n. 41, p. 87-105, 2020.

IIEG. Diagnóstico Municipal. Cuquío. Zapopan: Instituto de Información Estadística y Geográfica de Jalisco, 2019. Consultado el 6 de Enero 2021 en: https://iieg.gob.mx/ns/wp-content/uploads/2020/07/Cuqu\%C3\%ADo.pdf

INEGI. Censo de Población y Vivienda. México: Instituto Nacional de Estadística y Geografía, 2020. Consultado el 8 de Enero en: https://censo2020.mx/

KAY, Cristóbal. Transformaciones rurales en la era neoliberal. Dominio global del agro-negocio. Ecuador Debate, n. 106, p. 141-154, 2019.

KESSLER, Gabriel. La investigación social sobre juventud rural en América Latina. Estado de la cuestión de un campo en conformación. Revista Colombiana de Educación, n. 51, p. 16-39, 2006.

KESSLER, Gabriel. Juventud rural en América latina. Panorama de las investigaciones actuales. In: BRUNIARD, Rogelio. Educación, desarrollo rural y juventud. Buenos Aires: UNESCO; Secretaria de Agricultura, Ganaderia, pesca y alimentos, 2007. p. 16-67.

MESEGUER, Shantal. Imaginarios de futuro de la juventud rural. Educación Superior Intercultural en la Sierra de Zongolica, Veracrúz, México. Granada: Tesis Doctoral de la Universidad de Granada, 2012.

MORCH, Sven. Sobre el desarrollo y los problemas de la juventud. El surgimiento de la juventud como concepción sociohistórica. JOVENes: Revista de estudios sobre Juventud, v.1 p. 78-106, jul./set. 1996.

NU; CEPAL. Informe Regional de Población en América Latina y el Caribe 2011. Invertir en juventud. Santiago: CEPAL; UNFPA, 2012.

PACHECO, Lourdes. La construcción de cohesión social en la ruralidad. In: PACHECO, Lourdes; ROMÁN, Rosario; URTEAGA, Maritza. Jóvenes Rurales. Viejos dilemas, nuevas realidades. México: UAN; Juan Pablos, 2013. p. 19-30.

REGUILLO, Rossana. Culturas Juveniles: Formas Políticas del desencanto. 1. ed. Buenos Aires: Siglo XXI, 2012.

RIMISP. Informe Latinoamericano Pobreza y Desigualdad 2019. Juventud rural y territorio. Santiago: RIMISP; Centro Latinoamericano para el Desarrollo Rural, 2020.

SÁNCHEZ, David. Palos Altos entre la muchachada y la juventud: la condición juvenil rural en una comunidad ranchera de Jalisco. Asesor: Landázuri Benítez, Gisela. 2020. Tesis (Doctorado) - Universidad Autónoma Metropolitana, UAM Xochimilco, Ciudad de México, 2020.

SARAVÍ, Gozalo. Transiciones vulnerables. Juventud, desigualdad y exclusión en México. Ciudad de México: CIESAS; Casa Chata, 2009.

SOLOAGA, Isidro. Diagnóstico de las juventudes rurales de México. Serie documento de trabajo, n. 241. Programa Jóvenes Rurales, Territorios y Oportunidades: una estrategia de diálogos de políticas. Santiago, Chile: RIMISP, 2018.

Data de submissão: $31 / 01 / 2021$

Data de aceite: $16 / 02 / 2021$ 